Revue des patrimoines

19 | 2012

Patrimoines et conservation préventive. Pratiques comparées et nouveaux enjeux

\title{
Les infestations des collections patrimoniales : goût des insectes et évaluation des risques
}

\section{Grazia Nicosia}

\section{(2) OpenEdition}

Journals

Édition électronique

URL : http://journals.openedition.org/insitu/9840

DOI : $10.4000 /$ insitu. 9840

ISSN : 1630-7305

Éditeur

Ministère de la culture

Référence électronique

Grazia Nicosia, «Les infestations des collections patrimoniales : goût des insectes et évaluation des risques », In Situ [En ligne], 19 | 2012, mis en ligne le 27 septembre 2012, consulté le 19 avril 2019. URL : http://journals.openedition.org/insitu/9840 ; DOI : 10.4000/insitu.9840

Ce document a été généré automatiquement le 19 avril 2019

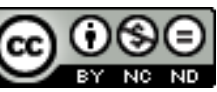

In Situ Revues des patrimoines est mis à disposition selon les termes de la licence Creative Commons Attribution - Pas d'Utilisation Commerciale - Pas de Modification 4.0 International. 


\title{
Les infestations des collections patrimoniales : goût des insectes et évaluation des risques
}

\author{
Grazia Nicosia
}

\section{Introduction}

1 Confronté à des infestations récurrentes, voire quasi chroniques, de certaines œuvres contemporaines, nous avons voulu identifier les facteurs qui interfèrent avec ces phénomènes. Ce problème est récurrent dans les réserves d'art contemporain et concerne un vaste corpus d'œuvres: Damien Hirst (1965-), Maurizio Cattelan (1960-), Annette Messager (1943-), Mark Dion (1961-), Huang Yong Ping (1954-), etc.

2 Les problèmes de conservation que soulèvent actuellement les œuvres contemporaines naturalia remontent à l'origine des collections. Au XVIII siècle, Georges-Louis Leclerc de Buffon (1707-1788) s'interrogeait déjà sur la préservation et le conditionnement des spécimens entomologiques du Cabinet du roi ${ }^{1}$. Les instituts d'art contemporain sont évidemment sensibilisés à la conservation des matériaux organiques comme le papier, le bois, les plumes, les textiles naturels, mais l'arrivée massive d'insectes séchés (Jan Fabre, (1958-)), de chocolat (Dieter Roth, (1930-1998)), ou des matières fécales (Gérard Gasiorowski, (1930-1996)) même patrimonialisées restent une denrée très attractive pour les insectes ravageurs, qui s'y installent naturellement et prolifèrent en masse.

Afin de comprendre les problèmes de conservation que pose ce type de biens culturels, nous avons réalisé un constat comparatif des pratiques patrimoniales relevant des traits typiques et variables suivant les thèmes des collections. Nous avons ainsi distingué ce qui est propre aux instituts d'art contemporain de ce qui dépend spécifiquement des œuvres.

4 Nous avons ainsi réalisé une enquête quantitative ${ }^{2} ; 121$ institutions y ont répondu ${ }^{3}$. L'échantillonnage concernait l'archéologie, l'art contemporain, l'art moderne, les arts et 
traditions populaires, les beaux-arts, l'ethnographie, les livres et les archives, le patrimoine industriel et technique, les sciences naturelles.

Nous avons élaboré un questionnaire comportant une soixantaine de questions classées en cinq parties. La première, « Informations générales », identifie les établissements et les individus qui ont répondu. La deuxième, "Infestations", relève les biens attractifs présents dans les collections, leurs conditions de conservation et de stockage, ainsi que l'appréhension des risques d'infestation, leurs détections et leurs traitements. La troisième, "Prévention", s'intéresse aux répulsifs et aux insecticides présents ou employés dans les institutions, ainsi qu'à la perception de leur toxicité et de leur efficacité. La quatrième, "Suivi, veille et monitorage", porte sur la surveillance et l'entretien ordinaire des collections. La cinquième permet enfin à ceux qui le souhaitent de laisser leurs coordonnées pour être informés ultérieurement des résultats de l'enquête.

6 Le questionnaire a été envoyé sous forme de lien ${ }^{4}$ par courriel à 600 institutions (musées, FRAC et centres d'art, muséums, archives...). Les réponses ont été directement enregistrées en ligne sur une période de 3 mois. 95 des 121 institutions, soit 78,5\%, ont répondu en totalité aux questions.

\section{Résultats 5}

7 Sur les 121 réponses, 101 émanent de musées et 11 de FRAC et centres d'art. $34 \%$ des musées ont une collection monothématique et $66 \%$ multithématique (tableau 1).

8 Les personnes qui ont répondu au questionnaire sont à $38 \%$ (46) des régisseurs, à $28,1 \%$ (34) des attachés de conservation et à 19,8\% (24) des conservateurs. Il s'agit en majorité de femmes - 61,5\% (72 femmes pour 45 hommes) - âgées en moyenne de 30 à 45 ans. $70,2 \%$ (85) ont moins de 15 ans d'expérience et $23,9 \%$ (27) déclarent n'avoir jamais reçu de formation spécifique à la régie des œuvres d'art.

Elles sont issues à 50 \% (57) d'une filière en histoire de l'art, à $15 \%$ (17) en histoire ou archéologie, à $12 \%$ (14) en biologie et sciences de la nature et à $8 \%$ (9) en beaux-arts et arts plastiques. Ces orientations ont souvent conditionné le choix de leur institution. En effet, les personnes ayant reçu une formation artistique se retrouvent à $23 \%$ dans des institutions d'art contemporain et à $16 \%$ dans des institutions d'art moderne; celles formées aux sciences de la nature sont à $40 \%$ dans des muséums d'histoire naturelle. L'histoire de l'art semble, en revanche, une formation moins déterminante, avec $18 \%$ des personnes dans des institutions de beaux-arts, $14 \%$ dans l'archéologie et $14 \%$ dans l'art contemporain. Il est important de noter que le service de régie est vacant pour 2,6 \% (3) des cas et ne comprend à $44,7 \%$ (51) qu'une seule personne.

Les établissements sont majoritairement - 67,8\% (82) - des petites et moyennes structures, avec des surfaces d'exposition inférieures à $1000 \mathrm{~m}^{2}$ et des réserves en deçà de $500 \mathrm{~m}^{2} .56,3 \%$ (67) ont des réserves externalisées. Les bâtiments d'exposition tiennent souvent de la demeure historique. $72 \%$ (79) sont antérieurs à 1900 et $27 \%$ (30) à 1700 . Dans 63 \% (59) des cas, les travaux de réfection datent de moins de 20 ans (fig. $\left.\mathbf{n}^{\circ} \mathbf{1}\right)$ (fig. $\mathbf{n}$ $\left.{ }^{\circ} 2\right)$. 
Figure 1

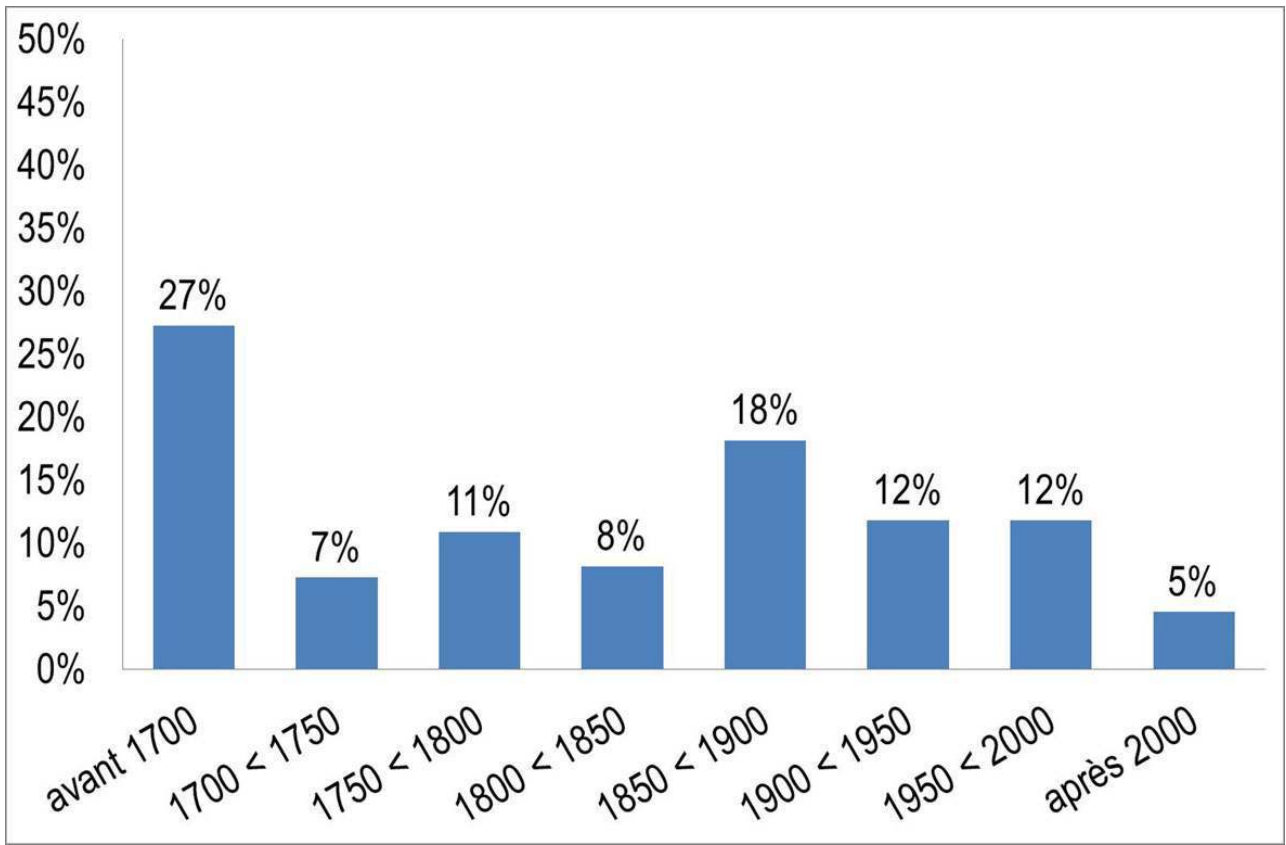

Date de construction du lieu d'exposition. $63 \%$ ont subi une réfection il y a moins de 20 ans.

(c) Grazia Nicosia.

Figure 2

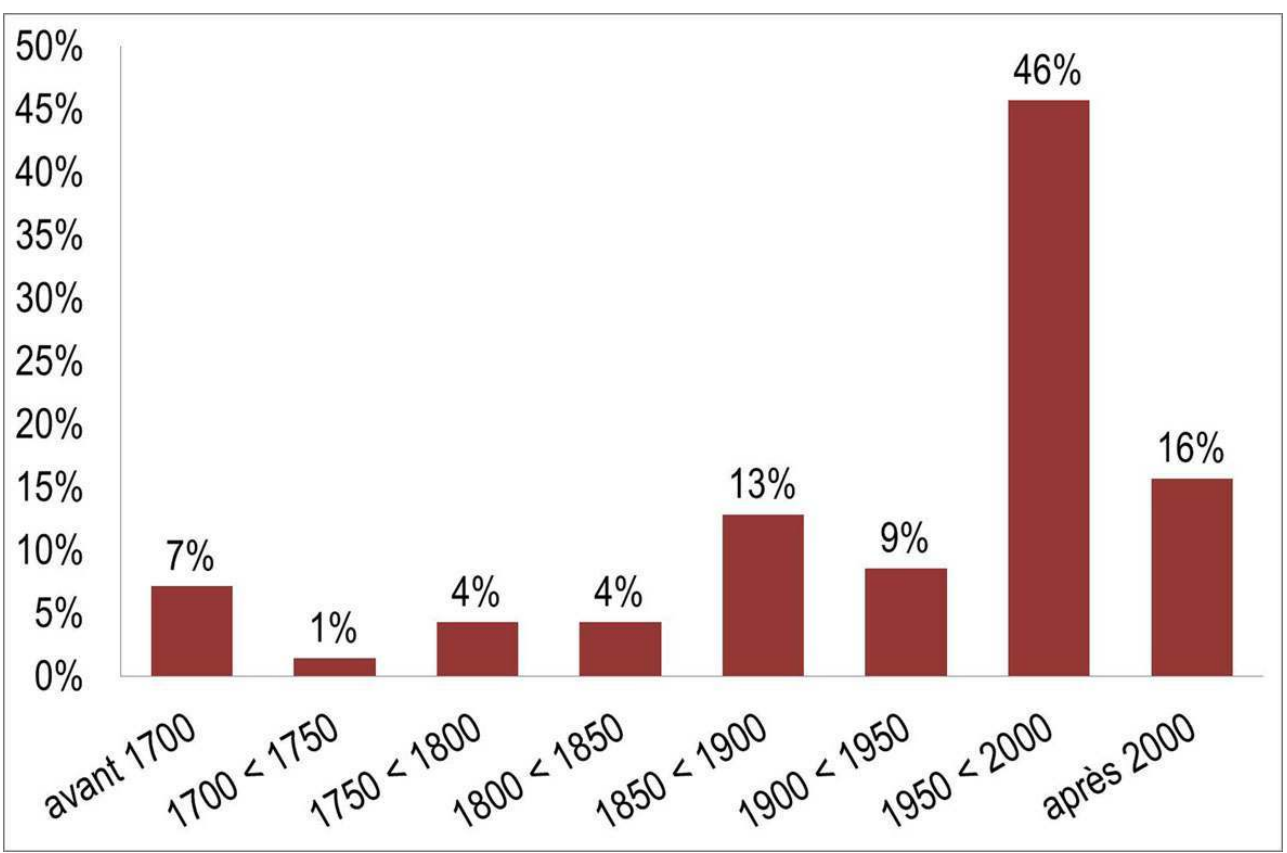

Date de construction des réserves. $58 \%$ ont bénéficié d'une réfection il y a moins de 20 ans.

(c) Grazia Nicosia.

11 Après avoir cerné les individus et les établissements, nous nous sommes intéressés plus particulièrement aux infestations. Dans 79,5\% (58) des cas, elles sont décelées au vu des dommages observés sur le bien, et dans 57,5\% (42) des cas par la présence d'insectes ou d'exuvies. Elles sont détectées à $68,5 \%$ (50) lors d'une vérification périodique de l'état 
sanitaire de la collection, à $15,1 \%$ (11) lors d'un constat de vérification pour prêt, et à $16,4 \%$ (12) par hasard. 70,3\% (52) des personnes ayant répondu déclarent connaître ou avoir fait identifier les insectes ravageurs, 53 ont précisé l'espèce : 72 entrées ont ainsi été reportées dans le tableau 6.

Il nous a semblé intéressant de recouper certaines questions avec les thématiques des collections afin de voir s'il y avait ou non corrélation. Comme la plupart des collections sont multithématiques, nous avons donc croisé chaque thème coché par les 121 institutions avec une vingtaine de questions concernant la gestion du climat, la prévention et la veille des risques, la nature et la récurrence des infestations, ainsi que les procédures et les traitements.

La première colonne du tableau 1 présente cette dernière répartition, la deuxième colonne quantifie le nombre de fois où le thème est cité (en moyenne quatre à cinq thèmes sont mentionnés par établissement, par exemple : archéologie, arts et traditions populaires, beaux-arts) (fig. $\left.\mathbf{n}^{\circ} \mathbf{3}\right)\left(\right.$ fig. $\left.\mathbf{n}^{\circ} \mathbf{4}\right)$.

Figure 3

\begin{tabular}{|c|c|c|c|c|}
\hline Institutions & \multicolumn{2}{|c|}{ Réponses } & \multicolumn{2}{|c|}{$\begin{array}{l}\text { Mention des thèmes dans les } \\
\text { institutions multithématiques }\end{array}$} \\
\hline $\begin{array}{c}\text { collections } \\
\text { multithématiques }\end{array}$ & 68 & $57 \%$ & & \\
\hline Archéologie & 6 & $5 \%$ & 55 & $14 \%$ \\
\hline Art contemporain & 11 & $9 \%$ & 46 & $12 \%$ \\
\hline Art moderne & 3 & $2 \%$ & 39 & $10 \%$ \\
\hline $\begin{array}{l}\text { Arts et traditions } \\
\text { populaires }\end{array}$ & 2 & $2 \%$ & 53 & $13 \%$ \\
\hline Beaux-arts & 7 & $6 \%$ & 65 & $16 \%$ \\
\hline Ethnographie & 2 & $2 \%$ & 48 & $12 \%$ \\
\hline Livres et archives & 1 & $1 \%$ & 35 & $9 \%$ \\
\hline $\begin{array}{l}\text { Patrimoine industriel } \\
\text { et technique }\end{array}$ & 3 & $2 \%$ & 26 & $7 \%$ \\
\hline Sciences naturelles & 9 & $7 \%$ & 29 & $7 \%$ \\
\hline Autres & 9 & $7 \%$ & & \\
\hline Réponses totales & 121 & & 396 & \\
\hline
\end{tabular}

Tableau 1 : Identification des collections monothématiques et répartition générale des thèmes.

(C) Grazia Nicosia. 


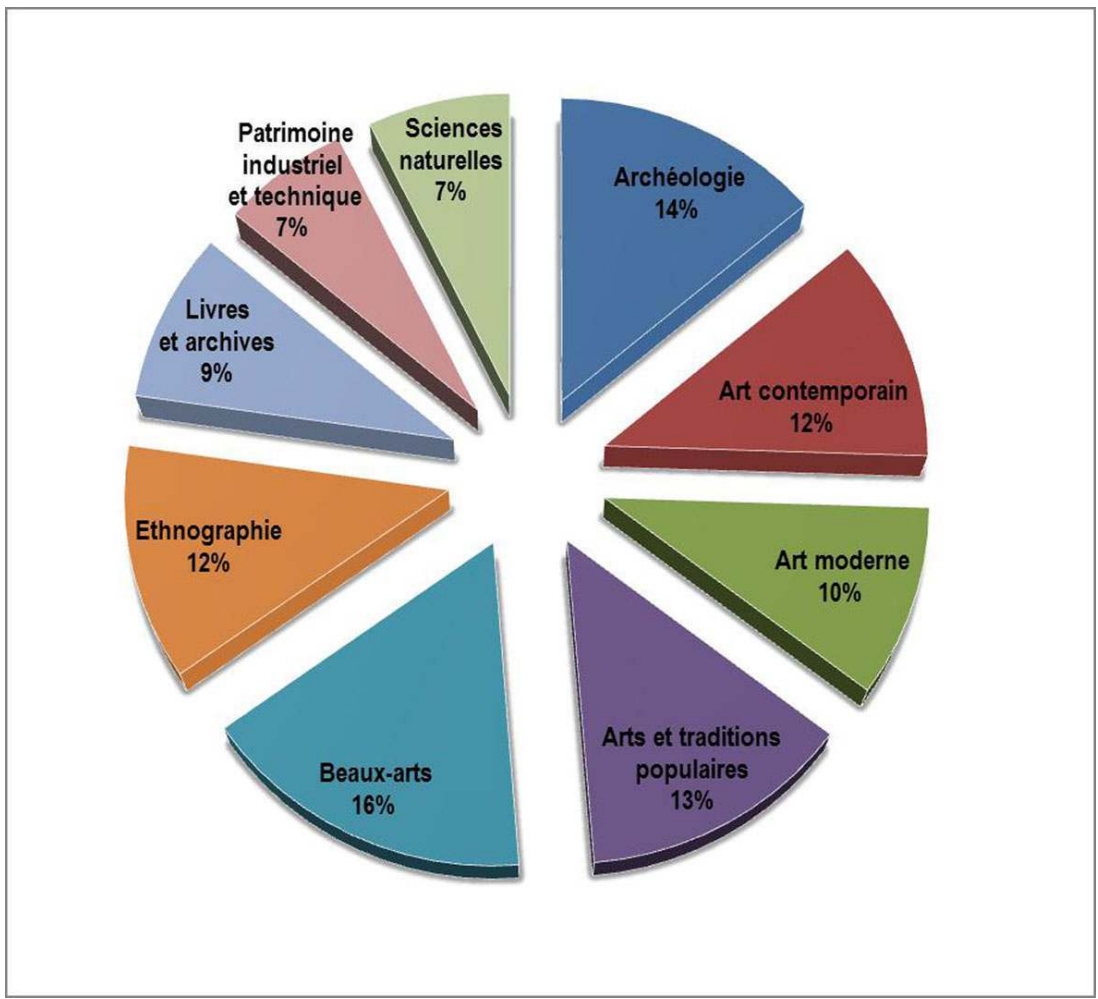

Répartition des collections dans les institutions multithématiques.

(c) Grazia Nicosia.

14 Les tableaux 2 et 3 présentent la gestion du climat et sa mesure dans les salles d'exposition et les réserves. La ligne «Réponses totales » comptabilise toujours les réponses par établissement, tandis que les colonnes «Réponses» dénombrent les réponses par thème, sachant qu'une même institution peut en cocher plusieurs (fig. $\mathbf{n}^{\circ} 5$ ) (fig. $\left.n^{\circ} 6\right)$ (fig. $\left.n^{\circ} 7\right)$ (fig. $n^{\circ} 8$ ). 
Figure 5

\begin{tabular}{|c|c|c|c|c|c|c|c|c|c|c|c|c|}
\hline & \multicolumn{3}{|c|}{$\begin{array}{l}\text { 1-Possédez-vous } \\
\text { un système derégulation } \\
\text { delacimatisation? }\end{array}$} & \multicolumn{3}{|c|}{$\begin{array}{l}\text { 2-Sioui, } \\
\text { esti régulèrement vérié } \\
\text { etentretenu? }\end{array}$} & \multicolumn{3}{|c|}{$\begin{array}{l}\text { 3-Possédez-vous } \\
\text { un système demesure } \\
\text { thermohygrométique? }\end{array}$} & \multicolumn{3}{|c|}{$\begin{array}{l}4-S i o u i \\
\text { est regulerrement relevé? }\end{array}$} \\
\hline & Rép. & $\mathrm{Non}$ & $a_{i}$ & Rép. & $\mathrm{Non}$ & $a_{i}$ & Rép. & Non & $a_{i}$ & Rép. & Non & $a_{i}$ \\
\hline \multirow{2}{*}{ Archéologie } & 53 & 29 & 24 & 29 & 7 & 22 & 55 & 12 & 43 & 47 & 9 & 38 \\
\hline & & $55 \%$ & $45 \%$ & & $24 \%$ & $76 \%$ & & $22 \%$ & $78 \%$ & & $19 \%$ & $81 \%$ \\
\hline \multirow{2}{*}{ Artcontemporain } & 41 & 17 & 24 & 27 & 4 & 23 & 44 & 9 & 35 & 39 & 8 & 31 \\
\hline & & $41 \%$ & $59 \%$ & & $15 \%$ & $85 \%$ & & $20 \%$ & $80 \%$ & & $21 \%$ & $79 \%$ \\
\hline \multirow{2}{*}{ Artmodeme } & 36 & 15 & 21 & 24 & 4 & 20 & 38 & 7 & 31 & 34 & 7 & 27 \\
\hline & & $42 \%$ & $58 \%$ & & $17 \%$ & $83 \%$ & & $18 \%$ & $82 \%$ & & $21 \%$ & $79 \%$ \\
\hline \multirow{2}{*}{ Arts et traditions populares } & 52 & 30 & 22 & 26 & 6 & 20 & 52 & 14 & 38 & 43 & 8 & 35 \\
\hline & & $58 \%$ & $42 \%$ & & $23 \%$ & $77 \%$ & & $27 \%$ & $73 \%$ & & $19 \%$ & $81 \%$ \\
\hline \multirow{2}{*}{ Beauxarts } & ๓3 & 33 & 30 & 33 & 6 & 27 & 65 & 9 & 56 & 60 & 10 & 50 \\
\hline & & $52 \%$ & $48 \%$ & & $18 \%$ & $82 \%$ & & $14 \%$ & $86 \%$ & & $17 \%$ & $83 \%$ \\
\hline \multirow{2}{*}{ Bhnographie } & 46 & 25 & 21 & 27 & 8 & 19 & 48 & 10 & 38 & 42 & 8 & 34 \\
\hline & & $54 \%$ & $46 \%$ & & $30 \%$ & $70 \%$ & & $21 \%$ & $79 \%$ & & $19 \%$ & $81 \%$ \\
\hline \multirow{2}{*}{ Lives etarchives } & 34 & 17 & 17 & 18 & 5 & 13 & 35 & 4 & 31 & 32 & 4 & 28 \\
\hline & & $50 \%$ & $50 \%$ & & $28 \%$ & $72 \%$ & & $11 \%$ & $89 \%$ & & $12 \%$ & $88 \%$ \\
\hline \multirow{2}{*}{ Patimoine industriel ettechnique } & 26 & 15 & 11 & 12 & 2 & 10 & 25 & 5 & 20 & 21 & 4 & 17 \\
\hline & & $58 \%$ & $42 \%$ & & $17 \%$ & $83 \%$ & & $20 \%$ & $80 \%$ & & $19 \%$ & $81 \%$ \\
\hline \multirow{2}{*}{ Sciences natureles } & 27 & 14 & 13 & 13 & 3 & 10 & 29 & 6 & 23 & 24 & 2 & 22 \\
\hline & & $52 \%$ & $48 \%$ & & $23 \%$ & $77 \%$ & & $21 \%$ & $79 \%$ & & $8 \%$ & $92 \%$ \\
\hline \multirow{2}{*}{ Réponses totales } & 117 & 66 & 51 & 61 & 14 & 47 & 119 & 27 & 92 & 99 & 17 & 82 \\
\hline & & $56 \%$ & $44 \%$ & & $23 \%$ & $77 \%$ & & $23 \%$ & $77 \%$ & & $17 \%$ & $83 \%$ \\
\hline
\end{tabular}

Tableau 2 : Gestion et mesure du climat des salles d'exposition.

(c) Grazia Nicosia.

Figure 6

\begin{tabular}{|c|c|c|c|c|c|c|c|c|c|c|c|c|}
\hline & \multicolumn{3}{|c|}{$\begin{array}{c}\text { 5-Possédezvous } \\
\text { unsystème derégulation } \\
\text { dela cimatisation? }\end{array}$} & \multicolumn{3}{|c|}{ 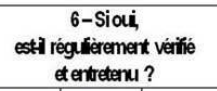 } & \multicolumn{3}{|c|}{$\begin{array}{l}\text { 7-Possédez-vous } \\
\text { unsysteme demesure } \\
\text { thermohygrométique? }\end{array}$} & \multicolumn{3}{|c|}{ 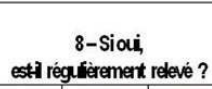 } \\
\hline & Rép. & Non & Oui & Rép. & Non & Oui & Rép. & Non & Oui & Rép. & Non & Oui \\
\hline \multirow{2}{*}{ Archéologie } & 53 & 27 & 26 & 32 & 8 & 24 & 54 & 19 & 35 & 37 & 6 & 31 \\
\hline & & $51 \%$ & $49 \%$ & & $25 \%$ & $75 \%$ & & $35 \%$ & $65 \%$ & & $16 \%$ & $84 \%$ \\
\hline \multirow{2}{*}{ Art contemporain } & 42 & 16 & 26 & 29 & 4 & 25 & 44 & 6 & 38 & 38 & 4 & 34 \\
\hline & & $38 \%$ & $62 \%$ & & $14 \%$ & $86 \%$ & & $14 \%$ & $86 \%$ & & $11 \%$ & $89 \%$ \\
\hline \multirow{2}{*}{ Artmodeme } & 37 & 13 & 24 & 28 & 5 & 23 & 39 & 9 & 30 & 30 & 4 & 26 \\
\hline & & $35 \%$ & $65 \%$ & & $18 \%$ & $82 \%$ & & $23 \%$ & $\pi \%$ & & $13 \%$ & $87 \%$ \\
\hline \multirow{2}{*}{ Arts etractions populares } & 52 & 31 & 21 & 27 & 8 & 19 & 53 & 17 & 36 & 38 & 6 & 32 \\
\hline & & $60 \%$ & $40 \%$ & & $30 \%$ & $70 \%$ & & $32 \%$ & $68 \%$ & & $16 \%$ & $84 \%$ \\
\hline \multirow{2}{*}{ Beackats } & 63 & 28 & 35 & 38 & 6 & 32 & 64 & 14 & 50 & 51 & 7 & 44 \\
\hline & & $44 \%$ & $56 \%$ & & $16 \%$ & $84 \%$ & & $22 \%$ & $78 \%$ & & $14 \%$ & $86 \%$ \\
\hline \multirow{2}{*}{ Ettnographie } & 46 & 22 & 24 & 30 & 8 & 22 & 48 & 14 & 34 & 35 & 5 & 30 \\
\hline & & $48 \%$ & $52 \%$ & & $27 \%$ & $73 \%$ & & $29 \%$ & $71 \%$ & & $14 \%$ & $86 \%$ \\
\hline \multirow{2}{*}{ Lives et archives } & 34 & 17 & 17 & 17 & 3 & 14 & 34 & 7 & 27 & 26 & 2 & 24 \\
\hline & & $50 \%$ & $50 \%$ & & $18 \%$ & $82 \%$ & & $21 \%$ & $79 \%$ & & $8 \%$ & $92 \%$ \\
\hline \multirow{2}{*}{ Patumoine incustriel at techrique } & 26 & 12 & 14 & 16 & 3 & 13 & 25 & 7 & 18 & 20 & 3 & 17 \\
\hline & & $46 \%$ & $54 \%$ & & $19 \%$ & $81 \%$ & & $28 \%$ & $72 \%$ & & $15 \%$ & $85 \%$ \\
\hline \multirow{2}{*}{ Sciences natureles } & 27 & 9 & 18 & 18 & 1 & 17 & 29 & 5 & 24 & 23 & 3 & 20 \\
\hline & & $33 \%$ & $67 \%$ & & $6 \%$ & $94 \%$ & & $17 \%$ & $83 \%$ & & $13 \%$ & $87 \%$ \\
\hline \multirow{2}{*}{ Réponses totales } & 118 & 59 & 59 & 70 & 14 & 56 & 119 & 32 & 87 & 92 & 14 & 78 \\
\hline & & $50 \%$ & $50 \%$ & & $20 \%$ & $80 \%$ & & $27 \%$ & $73 \%$ & & $15 \%$ & $85 \%$ \\
\hline
\end{tabular}

Tableau 3 : Gestion et mesure du climat des espaces de réserves.

(c) Grazia Nicosia. 
Figure 7

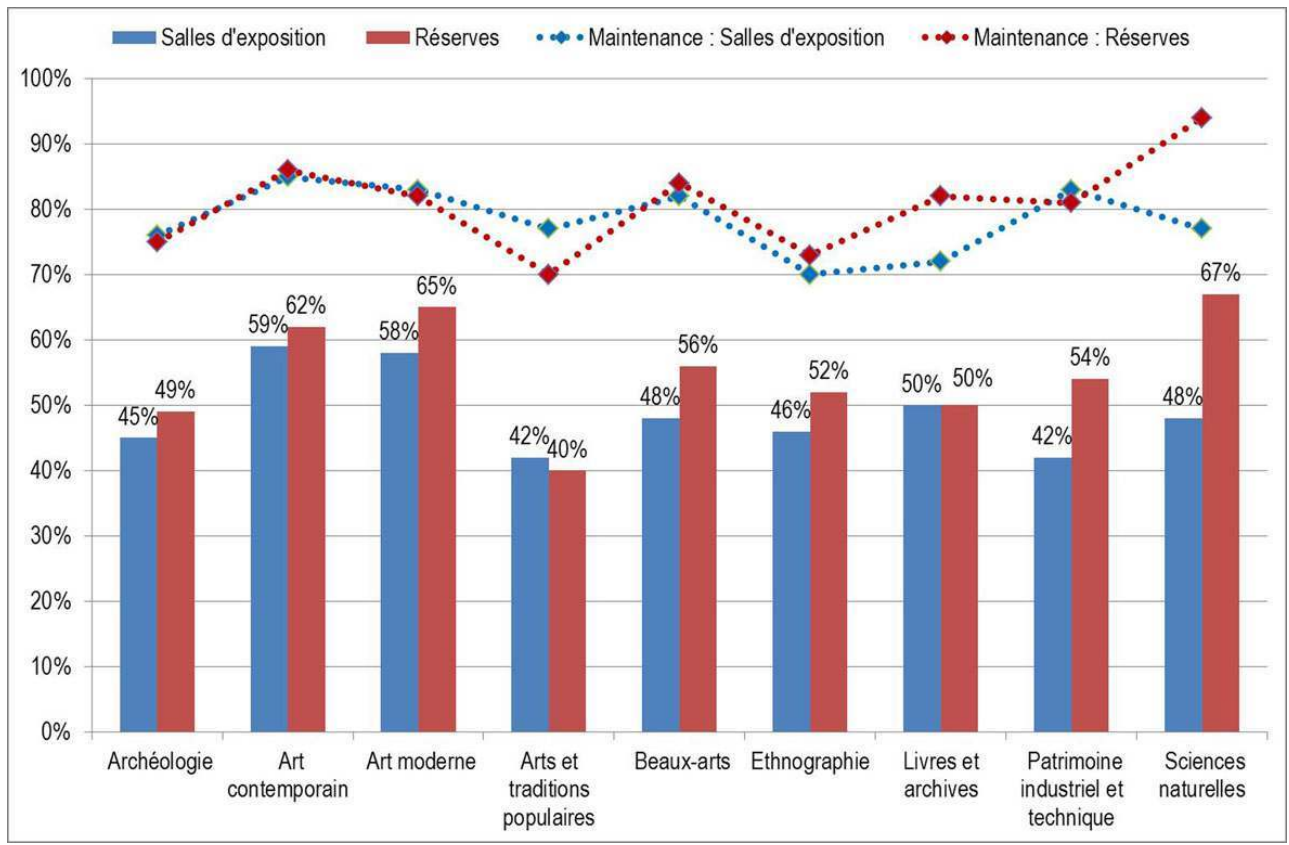

Figure 4 : Pourcentage des équipements de gestion du climat dans les réserves et salles d'exposition et leur maintenance régulière.

(c) Grazia Nicosia

\section{Figure 8}

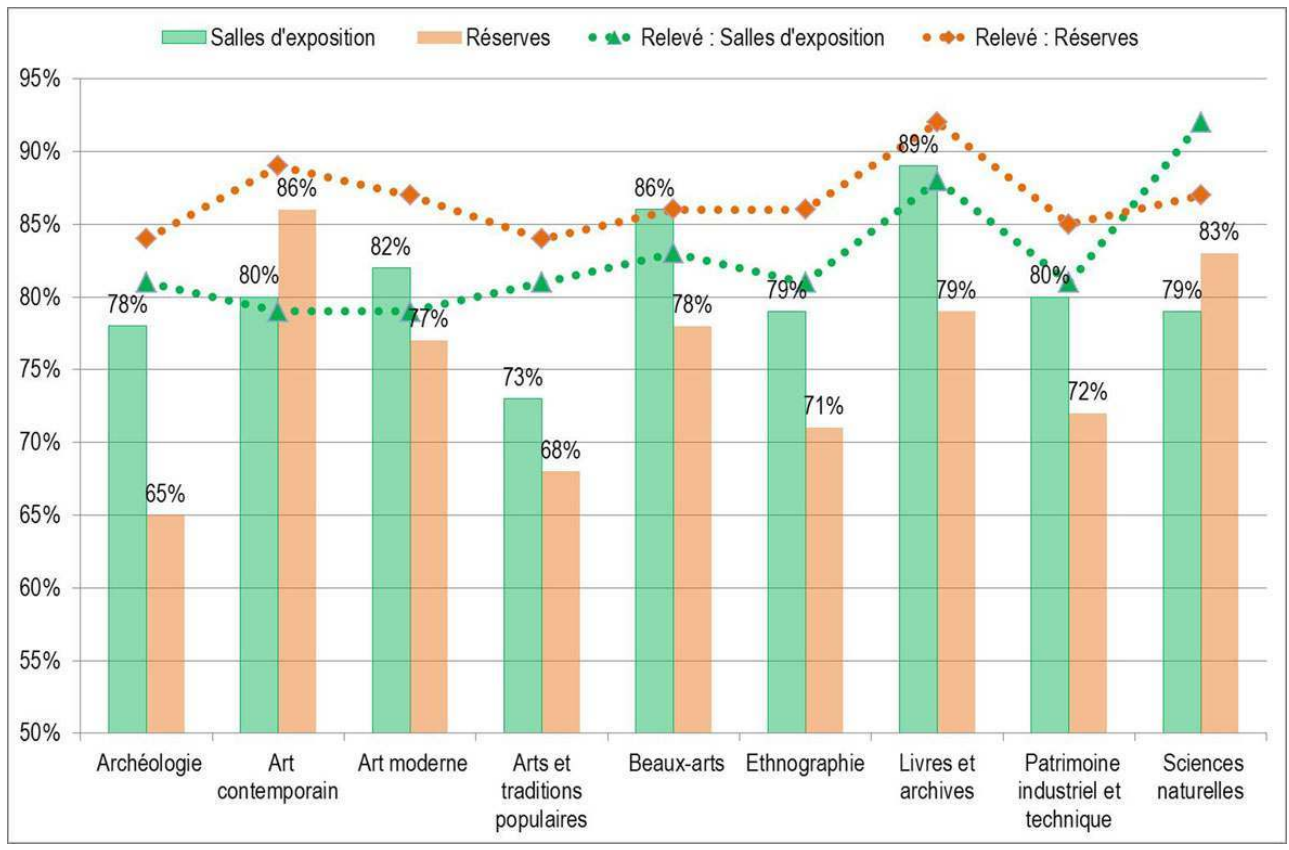

Figure 5 : Pourcentage des équipements thermohygrométriques dans les réserves et salles d'exposition et leur relevé régulier

(c) Grazia Nicosia. 
15 Le tableau 4 permet d'appréhender la perception du risque d'infestation des œuvres sensibles aux ravageurs, ainsi que les dispositifs de surveillance et de mise à distance des insectes (fig. $\left.\mathbf{n}^{\circ} \mathbf{9}\right)$.

Figure 9

\begin{tabular}{|c|c|c|c|c|c|c|c|c|c|}
\hline & \multicolumn{3}{|c|}{$\begin{array}{l}\text { 9-Certaines œuvres sontelles identifiées } \\
\text { comme présentant des risques vis-à-vis } \\
\text { des insectes ravageurs du patimoine? }\end{array}$} & \multicolumn{3}{|c|}{$\begin{array}{c}\text { 10-Avez-vous un moyen de prévention } \\
\text { pourtenir à distance } \\
\text { les insectes nuisibles? }\end{array}$} & \multicolumn{3}{|c|}{$\begin{array}{l}\text { 11-Avez-vous installé un dispositif } \\
\text { particulier de surveillance } \\
\text { des infestations? }\end{array}$} \\
\hline & Rép. & Non & $a_{i}$ & Rép. & Non & $a_{i}$ & Rép. & Non & $a_{i}$ \\
\hline \multirow{2}{*}{ Archéologie } & 35 & 7 & 28 & 43 & 35 & 8 & 41 & 27 & 14 \\
\hline & & $20 \%$ & $80 \%$ & & $81 \%$ & $19 \%$ & & $66 \%$ & $34 \%$ \\
\hline \multirow{2}{*}{ Artcontemporain } & 33 & 7 & 26 & 34 & 27 & 7 & 32 & 18 & 14 \\
\hline & & $21 \%$ & $79 \%$ & & $79 \%$ & $21 \%$ & & $56 \%$ & $44 \%$ \\
\hline \multirow{2}{*}{ Antmodeme } & 24 & 6 & 18 & 25 & 18 & 7 & 23 & 15 & 8 \\
\hline & & $25 \%$ & $75 \%$ & & $72 \%$ & $28 \%$ & & $65 \%$ & $35 \%$ \\
\hline \multirow{2}{*}{ Auts ettradtions populaires } & 38 & 7 & 31 & 44 & 36 & 8 & 44 & 27 & 17 \\
\hline & & $18 \%$ & $82 \%$ & & $82 \%$ & $18 \%$ & & $61 \%$ & $39 \%$ \\
\hline \multirow{2}{*}{ Beauxzrts } & 43 & 9 & 34 & 54 & 42 & 12 & 51 & 32 & 19 \\
\hline & & $21 \%$ & $79 \%$ & & $78 \%$ & $22 \%$ & & ๔\% & $37 \%$ \\
\hline \multirow{2}{*}{ Ethnographie } & 37 & 9 & 28 & 40 & 29 & 11 & 40 & 23 & 17 \\
\hline & & $24 \%$ & $76 \%$ & & $73 \%$ & $27 \%$ & & $58 \%$ & $42 \%$ \\
\hline \multirow[b]{2}{*}{ Livresetarchives } & 25 & 3 & 22 & 31 & 24 & 7 & 30 & 18 & 12 \\
\hline & & $12 \%$ & $88 \%$ & & $77 \%$ & $23 \%$ & & $60 \%$ & $40 \%$ \\
\hline \multirow{2}{*}{ Patrimoine industiel ettechnique } & 22 & 5 & 17 & 23 & 14 & 9 & 22 & 9 & 13 \\
\hline & & $23 \%$ & $77 \%$ & & $61 \%$ & $39 \%$ & & $41 \%$ & $59 \%$ \\
\hline \multirow{2}{*}{ Sciencesnaturelles } & 24 & 4 & 20 & 23 & 15 & 8 & 23 & 15 & 8 \\
\hline & & $17 \%$ & $83 \%$ & & $65 \%$ & $35 \%$ & & $65 \%$ & $35 \%$ \\
\hline \multirow{2}{*}{ Réponsestotales } & 85 & 19 & 66 & 97 & 74 & 23 & 94 & 57 & 37 \\
\hline & & $22 \%$ & $78 \%$ & & $76 \%$ & $24 \%$ & & $61 \%$ & $39 \%$ \\
\hline
\end{tabular}

Tableau 4 : Appréhension, prévention et veille des infestations.

(c) Grazia Nicosia.

Le tableau 5 différencie les attaques ponctuelles des infestations générales de collection et identifie le taux de diagnostic post-infestation pratiqué (fig. $\left.\mathbf{n}^{\circ} \mathbf{1 0}\right)$ (fig. $\left.\mathbf{n}^{\circ} \mathbf{1 1}\right)$.

Figure 10

\begin{tabular}{|c|c|c|c|c|c|c|c|c|c|}
\hline & \multicolumn{3}{|c|}{$\begin{array}{l}\text { 12-Les collections ontelles été } \\
\text { victimes d'attaques } \\
\text { d'insectes ravageurs? }\end{array}$} & \multicolumn{3}{|c|}{$\begin{array}{l}\text { 13-Certaines œuvres ontelles été } \\
\text { victimes d'attaques } \\
\text { d'insectes ravageurs? }\end{array}$} & \multicolumn{3}{|c|}{$\begin{array}{c}\text { 14-Avez-vous réalisé un diagnostic } \\
\text { préalable détaillé avec lidentification } \\
\text { de(s) l'espèce(s) et l'étendue de linfestation? }\end{array}$} \\
\hline & Rép. & Non & $a_{i}$ & Rép. & Non & $a_{i}$ & Rép. & Non & $a_{i}$ \\
\hline \multirow{2}{*}{ Archéologie } & 35 & 17 & 18 & 38 & 10 & 28 & 37 & 21 & 16 \\
\hline & & $49 \%$ & $51 \%$ & & $26 \%$ & $74 \%$ & & $57 \%$ & $43 \%$ \\
\hline \multirow{2}{*}{ Ait contemporain } & 27 & 17 & 10 & 31 & 11 & 20 & 28 & 17 & 11 \\
\hline & & ๔3\% & $37 \%$ & & $35 \%$ & $65 \%$ & & $61 \%$ & $39 \%$ \\
\hline \multirow[b]{2}{*}{ Aitmodeme } & 22 & 9 & 13 & 23 & 6 & 17 & 22 & 15 & 7 \\
\hline & & $41 \%$ & $59 \%$ & & $26 \%$ & $74 \%$ & & $68 \%$ & $32 \%$ \\
\hline \multirow{2}{*}{ Auts ettradlions populaires } & 37 & 14 & 23 & 36 & 10 & 26 & 40 & 22 & 18 \\
\hline & & $38 \%$ & $62 \%$ & & $28 \%$ & $72 \%$ & & $55 \%$ & $45 \%$ \\
\hline \multirow{2}{*}{ Beauxats } & 39 & 21 & 18 & 42 & 10 & 32 & 44 & 24 & 20 \\
\hline & & $54 \%$ & $46 \%$ & & $24 \%$ & $76 \%$ & & $55 \%$ & $45 \%$ \\
\hline \multirow{2}{*}{ Ethnographie } & 32 & 11 & 21 & 33 & 6 & 27 & 36 & 21 & 15 \\
\hline & & $34 \%$ & $66 \%$ & & $18 \%$ & $82 \%$ & & $58 \%$ & $42 \%$ \\
\hline \multirow[b]{2}{*}{ Livres et archives } & 24 & 12 & 12 & 26 & 8 & 18 & 27 & 15 & 12 \\
\hline & & $50 \%$ & $50 \%$ & & $31 \%$ & $69 \%$ & & $56 \%$ & $44 \%$ \\
\hline \multirow{2}{*}{ Patrimoineindustrielettechnique } & 18 & 8 & 10 & 18 & 5 & 13 & 18 & 8 & 10 \\
\hline & & $44 \%$ & $56 \%$ & & $28 \%$ & $72 \%$ & & $44 \%$ & $56 \%$ \\
\hline \multirow{2}{*}{ Sciencesnaturelles } & 22 & 7 & 15 & 20 & 3 & 17 & 22 & 9 & 13 \\
\hline & & $32 \%$ & $68 \%$ & & $15 \%$ & $85 \%$ & & $41 \%$ & $59 \%$ \\
\hline \multirow{2}{*}{ Réponsestotales } & 82 & 42 & 40 & 82 & 28 & 54 & 78 & 41 & 37 \\
\hline & & $51 \%$ & $49 \%$ & & $34 \%$ & $66 \%$ & & $53 \%$ & $47 \%$ \\
\hline
\end{tabular}

Tableau 5 : Nature des infestations et diagnostic réalisé.

(c) Grazia Nicosia. 
Figure 11

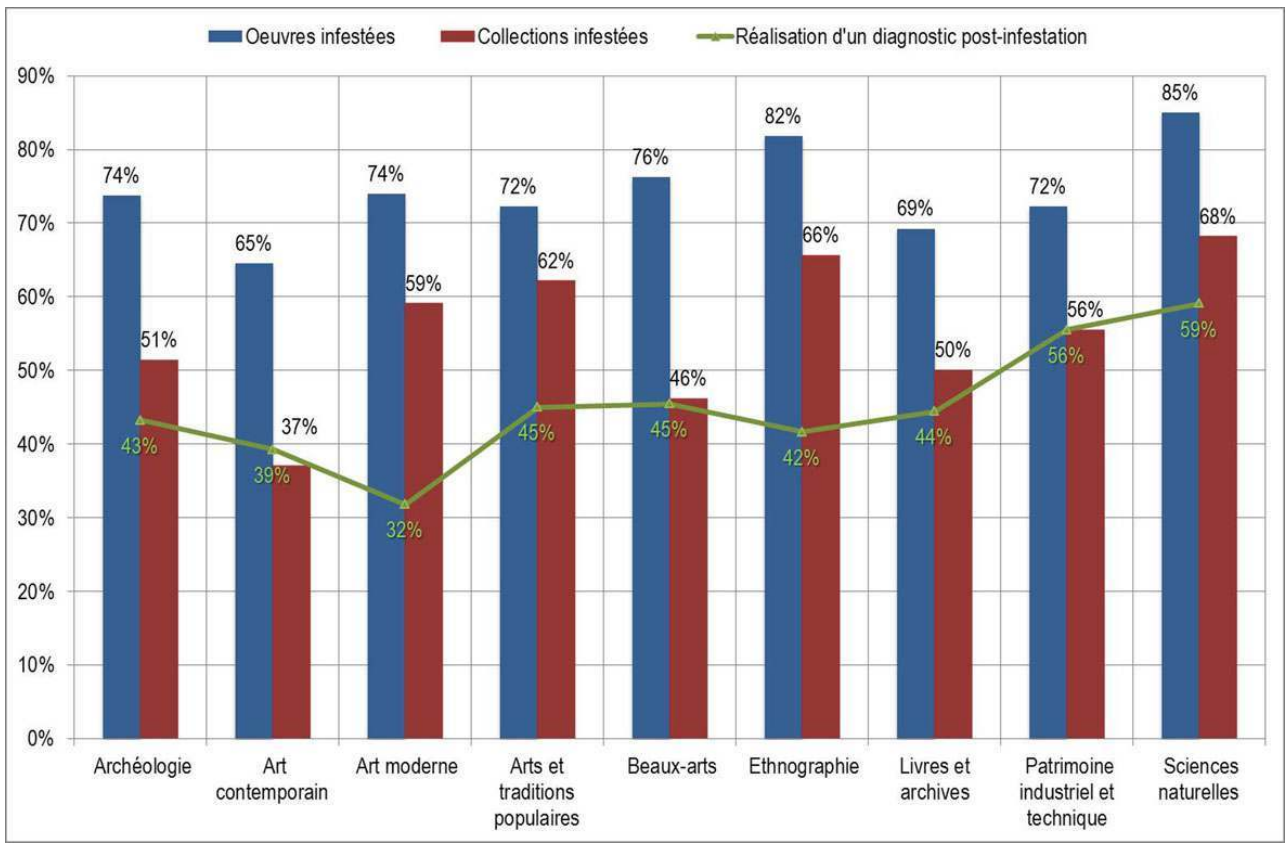

Figure 6 : Nature des infestations et diagnostic réalisé.

(c) Grazia Nicosia

Le tableau 6 mentionne les espèces recensées au sein des institutions et la récurrence des infestations (avec, entre parenthèses, le terme quand il est différent et sa fréquence) (fig. $\left.n^{\circ} 12\right)$ (fig. $\left.n^{\circ} 13\right)$. 
Figure 12

\begin{tabular}{|c|c|c|c|c|}
\hline & & & \multicolumn{2}{|c|}{$\begin{array}{c}\text { 15 - Les infestations sont-elles } \\
\text { un problème récurrent ? }\end{array}$} \\
\hline $\begin{array}{c}\text { Espèces } \\
\text { Vrillettes (15 vrillettes, 6 petites } \\
\text { vrillettes, 1 grosse villette) }\end{array}$ & 22 & $30,6 \%$ & 4 & 15 \\
\hline $\begin{array}{c}\text { Anthrènes (10 anthrènes et } \\
\text { 2 Anthrenus museorum) }\end{array}$ & 12 & $16,7 \%$ & 4 & 7 \\
\hline $\begin{array}{c}\text { Mites (7 mites et } \\
\text { 3 Tineola bisselliella) }\end{array}$ & 10 & $13,9 \%$ & 6 & 3 \\
\hline Stegobium paniceum & 5 & $6,9 \%$ & 2 & 3 \\
\hline Dermestes & 4 & $5,6 \%$ & & \\
\hline Xylophages & 4 & $5,6 \%$ & & \\
\hline Kératophages & 2 & $2,8 \%$ & & \\
\hline Capricorne & 2 & $2,8 \%$ & & \\
\hline Termites & 2 & $2,8 \%$ & & \\
\hline Attagènes & 2 & $2,8 \%$ & & \\
\hline $\begin{array}{c}\text { Lepisma saccharina } \\
\text { ou poisson d'argent }\end{array}$ & 2 & $2,8 \%$ & & \\
\hline Lasioderma serricorne & 1 & $1,4 \%$ & & \\
\hline Ptinidés & 1 & $1,4 \%$ & & \\
\hline Lépidoptères & 1 & $1,4 \%$ & & \\
\hline $\begin{array}{c}\text { Oligomerus } \\
\text { (ptilinoides et brunneus) }\end{array}$ & 2 & $2,8 \%$ & & \\
\hline Réponses totales & 72 & & 31 & \\
\hline
\end{tabular}

Tableau 6 : Espèces et récurrence des infestations.

(c) Grazia Nicosia.

\section{Figure 13}

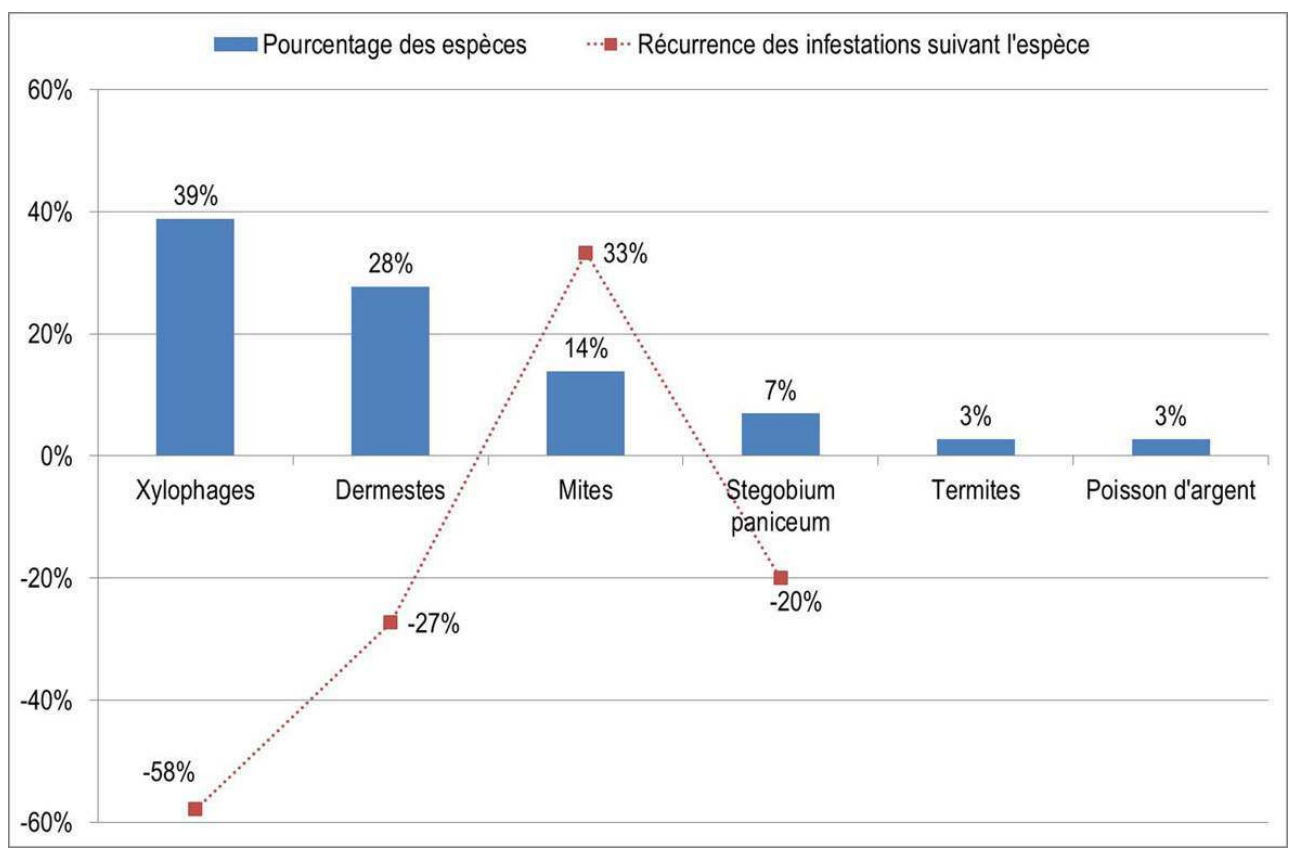

Figure 7 : Pourcentage des espèces citées et récurrences des infestations.

(c) Grazia Nicosia. 
Les tableaux $7^{6}$ et 8 permettent d'identifier l'espèce prédominante suivant le thème de l'institution (fig. $\left.\mathbf{n}^{\circ} 14\right)$ (fig. ${ }^{\circ}{ }^{\circ}$ ) (fig. $\left.n^{\circ} 16\right)$.

Figure 14

\begin{tabular}{|c|c|c|}
\hline Espèces & Institutions multithématiques & Institutions monothématiques \\
\hline Vrillettes & 18 & $\begin{array}{c}\text { 2 Beaux-arts } \\
\text { Art contemporain }\end{array}$ \\
\hline Anthrènes & 8 & 4 Sciences naturelles \\
\hline Mites & 7 & 3 Sciences naturelles \\
\hline $\begin{array}{c}\text { Stegobium } \\
\text { paniceum }\end{array}$ & 2 & $\begin{array}{c}\text { 2 Art contemporain } \\
\text { 1 Sciences naturelles }\end{array}$ \\
\hline
\end{tabular}

Tableau 7 : Répartition des espèces dans les institutions.

(c) Grazia Nicosia

Figure 15

\begin{tabular}{|c|c|c|c|c|c|c|c|c|}
\hline & \multicolumn{5}{|c|}{16 - Queles espèces avez-vous identifies ? } & \multicolumn{3}{|c|}{$\begin{array}{l}\text { 17-Les infestations de la collection sonkeles un } \\
\text { probleme recunert? }\end{array}$} \\
\hline & Réponses & Villettes & Anthrènes & Mines & $\begin{array}{l}\text { Stegobium } \\
\text { paniceum }\end{array}$ & Réponses & Non & Oui \\
\hline \multirow{2}{*}{ Archéologie } & 27 & 14 & 4 & 7 & 2 & 36 & 23 & 13 \\
\hline & & $16 \%$ & $11 \%$ & $19 \%$ & $6 \%$ & & $64 \%$ & $36 \%$ \\
\hline \multirow{2}{*}{ Art contemporain } & 14 & 5 & 1 & 4 & 4 & 29 & 19 & 10 \\
\hline & & $6 \%$ & $3 \%$ & $11 \%$ & $11 \%$ & & $66 \%$ & $34 \%$ \\
\hline \multirow{2}{*}{ Artmodeme } & 18 & 9 & 2 & 6 & 1 & 24 & 16 & 8 \\
\hline & & $10 \%$ & $6 \%$ & $17 \%$ & $3 \%$ & & $67 \%$ & $33 \%$ \\
\hline \multirow{2}{*}{$\begin{array}{l}\text { Aits ettradtions } \\
\text { populaires }\end{array}$} & 26 & 14 & 4 & 6 & 2 & 38 & 19 & 19 \\
\hline & & $16 \%$ & $11 \%$ & $17 \%$ & $6 \%$ & & $50 \%$ & $50 \%$ \\
\hline \multirow{2}{*}{ Beauc-arts } & 27 & 16 & 3 & 6 & 2 & 45 & 28 & 17 \\
\hline & & $18 \%$ & $8 \%$ & $17 \%$ & $6 \%$ & & $62 \%$ & $38 \%$ \\
\hline \multirow{2}{*}{ Ethnographie } & 26 & 12 & 6 & 6 & 2 & 39 & 22 & 17 \\
\hline & & $14 \%$ & $17 \%$ & $17 \%$ & $6 \%$ & & $56 \%$ & $44 \%$ \\
\hline \multirow{2}{*}{ Lives et archives } & 20 & 10 & 4 & 4 & 2 & 27 & 18 & 9 \\
\hline & & $11 \%$ & $11 \%$ & $11 \%$ & $6 \%$ & & $67 \%$ & $33 \%$ \\
\hline \multirow{2}{*}{$\begin{array}{l}\text { Patimoine incustriel } \\
\text { ettechrique }\end{array}$} & 13 & 5 & 4 & 4 & 0 & 20 & 14 & 6 \\
\hline & & $6 \%$ & $11 \%$ & $11 \%$ & $0 \%$ & & $70 \%$ & $30 \%$ \\
\hline \multirow{2}{*}{ Sciences natureles } & 17 & 2 & 8 & 6 & 1 & 21 & 8 & 13 \\
\hline & & $2 \%$ & $22 \%$ & $17 \%$ & $3 \%$ & & $38 \%$ & $62 \%$ \\
\hline \multirow{2}{*}{ Réponses totales } & 188 & 87 & 36 & 49 & 16 & 83 & 52 & 31 \\
\hline & & $46 \%$ & $19 \%$ & $26 \%$ & $9 \%$ & & $63 \%$ & $37 \%$ \\
\hline
\end{tabular}

Tableau 8 : Répartition générale des espèces suivant les thèmes des collections.

(c) Grazia Nicosia. 
Figure 16

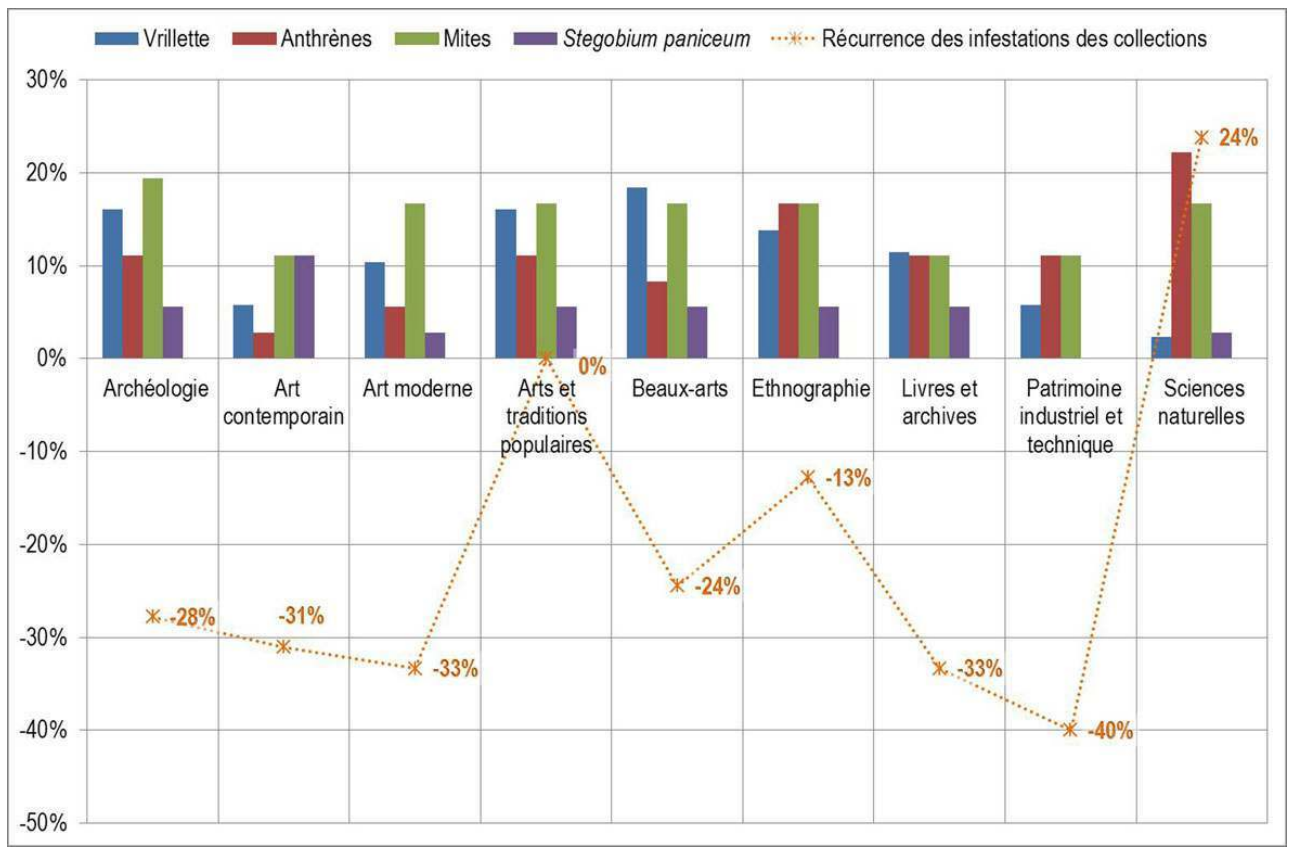

Figure 8 : Répartition des insectes par collection et récurrence des infestations en pourcentage.

(c) Grazia Nicosia

19 Le tableau 9 répertorie les procédures et les traitements suivant le type de collection (fig. $\left.n^{\circ} 17\right)$ (fig. $n^{\circ} 18$ ).

Figure 17

\begin{tabular}{|c|c|c|c|c|c|c|c|c|c|c|c|c|}
\hline & \multicolumn{3}{|c|}{$\begin{array}{l}\text { 18-Avez-vous une } \\
\text { procédure } \\
\text { en cas d'infestation? }\end{array}$} & \multicolumn{3}{|c|}{$\begin{array}{l}\text { 19-Avez-vous effectué } \\
\text { un traitement curatif } \\
\text { des biens culturels } \\
\text { infestés? }\end{array}$} & \multicolumn{3}{|c|}{$\begin{array}{l}\text { 20-Avez-vous effectué } \\
\text { un traitement insecticide } \\
\text { des lieux de stockage et } \\
\text { d'exposition? }\end{array}$} & \multicolumn{3}{|c|}{$\begin{array}{l}\text { 21-Le traitement de linfestation } \\
\text { attil été fait par un service inteme à } \\
\text { l'établissement? }\end{array}$} \\
\hline & Rép. & Non & $a_{i}$ & Rép. & Non & $a i$ & Rép. & Non & $a i$ & Rép. & Non & $\mathrm{Oi}_{i}$ \\
\hline \multirow{2}{*}{ Archéologie } & 46 & 20 & 26 & 34 & 2 & 32 & 24 & 10 & 14 & 30 & 15 & 15 \\
\hline & & $43 \%$ & $57 \%$ & & $6 \%$ & $94 \%$ & & $42 \%$ & $58 \%$ & & $50 \%$ & $50 \%$ \\
\hline \multirow{2}{*}{ Autcontemporain } & 37 & 18 & 19 & 23 & 3 & 20 & 20 & 10 & 10 & 21 & 13 & 8 \\
\hline & & $49 \%$ & $51 \%$ & & $13 \%$ & $87 \%$ & & $50 \%$ & $50 \%$ & & $62 \%$ & $38 \%$ \\
\hline \multirow{2}{*}{ Artmodeme } & 29 & 11 & 18 & 20 & 2 & 18 & 13 & 5 & 8 & 17 & 9 & 8 \\
\hline & & $38 \%$ & $62 \%$ & & $10 \%$ & $90 \%$ & & $38 \%$ & $62 \%$ & & $53 \%$ & $47 \%$ \\
\hline \multirow{2}{*}{ Aits ettraditionspopulares } & 46 & 21 & 25 & 36 & 5 & 31 & 30 & 13 & 17 & 33 & 17 & 16 \\
\hline & & $46 \%$ & $54 \%$ & & $14 \%$ & $86 \%$ & & $43 \%$ & $57 \%$ & & $52 \%$ & $48 \%$ \\
\hline \multirow{2}{*}{ Beaux-ats } & 55 & 20 & 35 & 42 & 4 & 38 & 31 & 12 & 19 & 39 & 20 & 19 \\
\hline & & $36 \%$ & $64 \%$ & & $10 \%$ & $90 \%$ & & $39 \%$ & $61 \%$ & & $51 \%$ & $49 \%$ \\
\hline \multirow{2}{*}{ Ethnographie } & 42 & 17 & 25 & 34 & 4 & 30 & 27 & 12 & 15 & 30 & 16 & 14 \\
\hline & & $40 \%$ & $60 \%$ & & $12 \%$ & $88 \%$ & & $44 \%$ & $56 \%$ & & $53 \%$ & $47 \%$ \\
\hline \multirow{2}{*}{ Livres et archives } & 32 & 14 & 18 & 22 & 1 & 21 & 18 & 8 & 10 & 23 & 10 & 13 \\
\hline & & $44 \%$ & $56 \%$ & & $5 \%$ & $95 \%$ & & $44 \%$ & $56 \%$ & & $43 \%$ & $57 \%$ \\
\hline \multirow{2}{*}{$\begin{array}{l}\text { Patrimoineindustielet } \\
\text { technique }\end{array}$} & 23 & 7 & 16 & 18 & 1 & 17 & 16 & 8 & 8 & 17 & 8 & 9 \\
\hline & & $30 \%$ & $70 \%$ & & $6 \%$ & $94 \%$ & & $50 \%$ & $50 \%$ & & $47 \%$ & $53 \%$ \\
\hline \multirow{2}{*}{ Sciencesnaturelles } & 25 & 7 & 18 & 22 & 1 & 21 & 20 & 5 & 15 & 22 & 8 & 14 \\
\hline & & $28 \%$ & $72 \%$ & & $5 \%$ & $95 \%$ & & $25 \%$ & $75 \%$ & & $36 \%$ & $64 \%$ \\
\hline \multirow{2}{*}{ Réponsestotales } & 104 & 45 & 59 & 72 & 6 & 66 & 56 & 22 & 34 & 68 & 34 & 34 \\
\hline & & $43 \%$ & $57 \%$ & & $8 \%$ & $92 \%$ & & $39 \%$ & $61 \%$ & & $50 \%$ & $50 \%$ \\
\hline
\end{tabular}

Tableau 9 : Procédures de traitement des infestations.

(c) Grazia Nicosia 
Figure 18

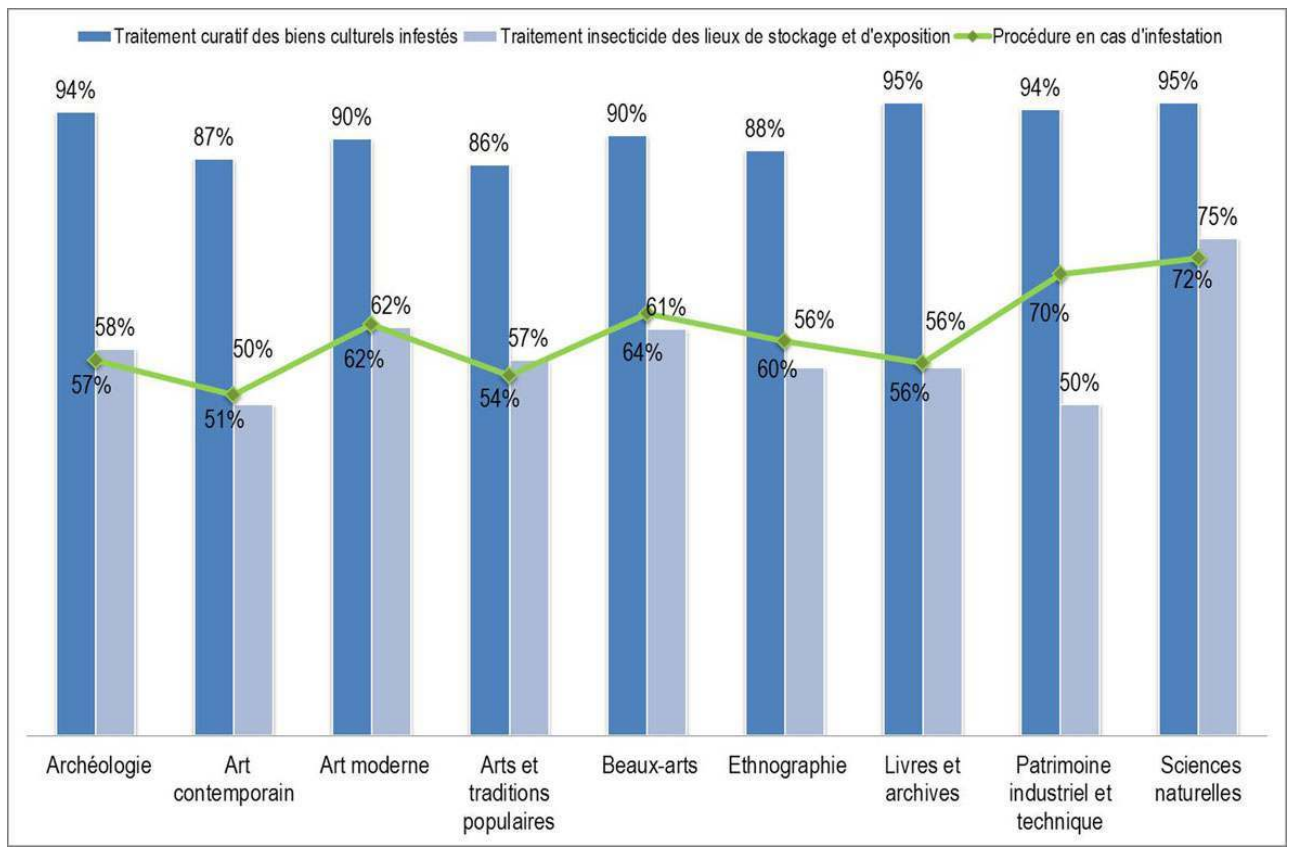

Figure 9 : Procédure et traitements réalisés en cas d'infestations déclarées en pourcentage. (c) Grazia Nicosia.

L'analyse des résultats pointe des corrélations factuelles entre les thèmes des institutions, la gestion de la climatisation, l'appréhension, la veille et la probabilité des infestations.

\section{Discussion}

Ces résultats mettent en évidence des disparités importantes de moyens, de prévention et de potentiel d'infestation, suivant le thème de la collection.

Les collections d'art contemporain et d'art moderne sont les plus équipées pour la régulation et la mesure du climat (tableaux 2 et 3). Le matériel est entretenu et les mesures sont régulièrement relevées. Les collections d'archéologie et celles d'arts et traditions populaires sont les moins équipées et les moins entretenues. Les collections de beaux-arts, d'ethnographie, de livres et d'archives ont des résultats proches de la moyenne, avec les particularités suivantes: celles de beaux-arts, de livres et d'archives sont plus équipées en matériel thermohygrométrique, mais les mesures sont plus régulièrement relevées que dans le dernier secteur. Les collections d'ethnographie sont celles qui entretiennent le moins la climatisation des salles et des réserves. Celles de sciences naturelles sont dans la moyenne pour l'équipement des salles d'exposition, mais les systèmes de régulation et de mesures sont très régulièrement entretenus et relevés. Leur spécificité tient à l'équipement des réserves, l'entretien et le relevé y étant nettement au-dessus de la moyenne des autres collections.

Les secteurs des sciences naturelles, des livres et des archives qui font partie de ceux qui mesurent et régulent le plus le climat sont également ceux qui appréhendent le plus les risques d'infestation (tableau 4). Les collections de sciences naturelles et de patrimoine industriel et technique sont majoritairement pourvues en moyens de prévention pour 
tenir à distance les insectes, mais les systèmes de surveillance d'infestation sont bien moins présents dans les collections de sciences naturelles, les plus équipées à cet égard étant celles d'art contemporain et celles du patrimoine industriel et technique. Les collections de sciences naturelles et d'ethnographie déclarent être les plus infestées, au niveau des collections mêmes comme au niveau des biens isolés. Les collections des beaux-arts sont surtout victimes d'attaques isolées. Quant à celles d'art contemporain, elles relèvent des pourcentages d'attaques de collections de biens culturels les plus bas. Il importe de noter qu'elles font partie, avec l'art moderne, des secteurs qui réalisent le moins de diagnostics lors des infestations (espèce, étendue), contrairement aux collections de patrimoine industriel et technique ou des sciences naturelles.

Sur les 72 insectes mentionnés, quatre espèces sont réellement identifiables par leur nombre et leur dénomination - la vrillette, l'anthrène, la mite et le Stegobium paniceum -, la plus citée étant la vrillette - 30 \% (22) -, suivie de l'anthrène (16,7 \%), de la mite (13,9 \%) et du Stegobium paniceum (6,9\%). Notons que la récurrence des infestations est majoritairement négative ( $76 \%$ ). Ce taux coïncide avec celui des relevés d'attaques d'anthrènes et de Stegobium paniceum, qui est, avec celui de la vrillette, le plus bas. Seule la mite se distingue par une récurrence d'infestation positive.

La vrillette semble préférer les collections multithématiques, avec un penchant pour les beaux-arts. L'anthrène et la mite sont proportionnellement plus présents dans les collections de sciences naturelles. Le Stegobium paniceum, quant à lui, semble préférer les collections d'art contemporain et de sciences naturelles.

Après infestation, les collections de sciences naturelles se démarquent à nouveau de celles du patrimoine industriel et technique : les procédures en cas d'infestation y étant supérieures de même que les traitements appliqués aux biens infestés et aux locaux. Les collections d'art contemporain et d'arts et traditions populaires sont celles où les biens et les locaux infestés sont les moins bien traités, les procédures en cas d'infestation étant également moindres. Il est à souligner qu'en général les traitements sont, à $50 \%$, effectués à l'extérieur. Seules les collections de sciences naturelles, de patrimoine industriel et technique, de livres et d'archives sont majoritairement traitées en interne.

\section{Les collections d'art contemporain}

De par leur nature, les œuvres d'art contemporain sont très attractives pour les insectes ravageurs et sont la plupart du temps infestées de manière cyclique. Les matériaux façonnés et assemblés par les artistes contemporains sont très variés, allant par exemple du pain (Raymond Waydelich, (1938-)) au chocolat (Dieter Roth, (1930-1998)), en passant par des insectes séchés (Jan Fabre) et des spécimens naturalisés (Maurizio Cattelan, (1960) ).

28 L'enquête montre l'attraction particulière que chaque type de collection exerce sur les insectes, un constat empirique qui permet de mieux cerner la spécificité de l'art contemporain vis-à-vis des infestations. Il en ressort que ces collections représentent $3,8 \%$ des institutions monothématiques et $11,6 \%$ des thèmes cités. Elles sont nettement plus équipées en climatisation et en thermohygrométrie, aussi bien dans les salles d'exposition que dans les réserves. Les biens sensibles aux ravageurs sont identifiés à $79 \%$, mais ces institutions disposent de moins de dispositifs répulsifs et de surveillance. 
il s'agisse d'infestations isolées ou d'infestations générales, les collections d'art contemporain sont moins attaquées que la moyenne, et de préférence par la mite et le Stegobium paniceum; la récurrence des infestations est légèrement au-dessous de la moyenne.

Bien que les institutions responsables réalisent majoritairement des diagnostics postinfestation, elles traitent bien moins les œuvres et les locaux infestés (figure 9).

31 collections d'art contemporain sont mieux équipées pour diagnostiquer que la plupart des institutions, mais elles utilisent peu les systèmes de mise à distance préventifs lorsque les attaques sont décelées, mais $13 \%$ des biens et $50 \%$ des locaux ne sont pas traités.

\section{Conclusions}

Ces résultats permettent non seulement de confirmer que certaines thématiques patrimoniales bénéficient de plus ou moins de moyens, mais également d'appréhender comment la structure institutionnelle conçoit la conservation des biens culturels et leurs agresseurs. Cette enquête pointe des pratiques endémiques aux types de collection et identifie des facteurs structurels et statutaires comme déterminants dans le processus des infestations. Suivant le type de collection, le régisseur aura une formation différente, les biens seront ou pas identifiés «à risques» avec les mesures qui s'en suivent. L'équipement de gestion et de contrôle du climat témoigne certes des moyens investis, mais nous avons noté que sa répartition dans les espaces de réserves ou de stockage reste spécifique. Outre l'attraction éprouvée par les ravageurs pour certains matériaux constitutifs, plusieurs d'entre eux manifestent un goût patrimonial particulier: la vrillette pour les collections multithématiques avec une prédilection pour les beaux-arts, ou l'anthrène et la mite pour celles de sciences naturelles. Le taux de réinfestations dépend d'un cumul de facteurs déterminants tels que la nature des collections, les traitements effectués, mais également les espèces considérées.

Il n'existe pas de solution exhaustive garantissant la protection des biens culturels contre les ravageurs, une seule action ne pouvant venir à bout du problème. Depuis une trentaine d'années, le domaine patrimonial ${ }^{7}{ }^{8}$ 'inspire des stratégies intégrées de lutte contre les nuisibles, l'Integrated Pest Management (IPM), utilisées notamment dans les domaines phytosanitaire et agroalimentaire. L'infestation est recontextualisée et ses facteurs critiques sont traités simultanément. Ils coordonnent conjointement les informations et les actions sur les ravageurs et l'environnement, afin de contrôler les dégradations, en deçà d'un seuil jugé inacceptable, par les moyens les plus économiques, avec le moins de risques possible pour les personnes, les biens et le milieu'.

S'il est important d'identifier les ravageurs et la nature de la collection, il s'avère également primordial de connaître l'environnement et le fonctionnement de l'institution et du personnel. Cette première synthèse nous a permis d'évaluer les moyens et les stratégies des institutions patrimoniales, ainsi que les vulnérabilités des biens culturels et leurs espèces nuisibles. Notre investigation continue en 2012 afin d'affiner cette étude systémique des infestations, de hiérarchiser et quantifier les facteurs déterminants permettant une évaluation des risques spécifiques aux infestations du patrimoine. 


\section{NOTES}

1. - «Les pièces qui sont desséchées demandent encore un plus grand soin; les insectes qui y naissent et qui y trouvent leur aliment les détruisent dans l'intérieur avant qu'on les ait aperçus ; il y a des vers, des scarabées, des teignes, des papillons, des mites, etc. [...] Tous ces insectes pullulent en peu de temps, et leur génération est si abondante que le nombre en deviendrait prodigieux, si on n'employait pas différents moyens pour les détruire.» LECLERC BUFFON, Georges-Louis, DAUBENTON, Louis-Jean-Marie, DESMAREST, Anselme-Gaëtan. CEuvres complètes de Buffon, avec les descriptions anatomiques de Daubenton, son collaborateur. Paris : Verdière et Ladrange, 1824-1832, tome 2 (1763), p. 163.

2. - Étude réalisée avec le soutien du CNAP, centre national des arts plastiques, ministère de la Culture et de la Communication, allocation de recherche en restauration.

3. - NICOSIA, Grazia. "Which strategies are used against insect pests : a survey in 120 institutions of public heritage". Journal of Entomological and Acarological Research (JEAR), 2011, Università di Milano, 2011, Vol 43, n² 2, p. 137-148.

4. - Voir le site : http://www.surveymonkey.com/s/insectes-du-patrimoine.

5. - NICOSIA, Grazia. Conserver les œuvres contemporaines naturalia - Étude des œuvres entomologiques de Jan Fabre. Conservation-restauration. Paris : Centre national des arts plastiques, 2012.

6. - Les espèces citées une seule fois (tableau 6) ne sont pas représentées. Xylophages = Vrillettes + Xylophages + Capricornes. Dermestes $=$ Anthrènes + Dermestes + Kératophages.

7. - PINNIGER, David. Integrated Pest Management in Museums, Archives and Historic Houses. Londres : Archetype Publications, 2004.

8. - STRANG, Tom, KIGAWA, Rika. «La lutte contre les parasites des biens culturels ». Bulletin technique de l'ICC, 2009, n² 29, p. 1-47.

9. - DHANG, Partho. Urban Pest Management : An Environmental Perspective. Oxfordshire : CABI Publishing, 2011, p. 218.

\section{RÉSUMÉS}

Cette étude met en évidence des disparités importantes de moyens, de prévention et de potentiel d'infestation suivant le thème de la collection. Les insectes ravageurs semblent également faire cette différenciation institutionnelle. Ces résultats permettent d'identifier de nouveaux facteurs déterminants dans les infestations des collections patrimoniales en intégrant aux facteurs biologiques et environnementaux ceux statutaires du bien culturel et structurel de l'institution.

\section{INDEX}

Mots-clés : enquête, infestation, collection, bien culturel, naturalia, ravageurs du patrimoine, stratégie, lutte intégrée, IPM, facteur déterminant, évaluation des risques, prévention des risques, conservation préventive, traitement insecticide 


\section{AUTEUR}

GRAZIA NICOSIA

Consultante en conservation préventive, Conservation - Restauration d'œuvres peintes et art contemporain grazia.nicosia@gmail.com 